\title{
Cost-benefit analysis of screening for diabetic retinopathy among Chinese type 2 diabetes: decision from different perspectives of a population-based study in Kinmen, Taiwan
}

\author{
Ching-Ching Peng ${ }^{1}$, Pesus Chou ${ }^{2}$, Tao-Hsin Tung ${ }^{1,3}$, Wu-Hsiung Chien ${ }^{1}$, Fenq-Lih Lee ${ }^{4}$, Shih-Jen \\ Chen ${ }^{4}$, Shih-Tzer Tsai ${ }^{5}$, Chi-Ming Liu ${ }^{1,2}$, Jorn-Hon Liu ${ }^{1,6^{*}}$
}

${ }^{1}$ Cheng Hsin General Hospital, Taipei, Taiwan, China; ch2876@chgh.org.tw

${ }^{2}$ Community Medicine Research Center \& Institute of Public Health, National Yang-Ming University, Taipei, Taiwan, China

${ }^{3}$ Faculty of Public Health, School of Medicine, Fu-Jen Catholic University, Taipei, Taiwan, China

${ }^{4}$ Department of Ophthalmology, Veterans General Hospital, Taipei, Taiwan, China

${ }^{5}$ Department of Medicine, Veterans General Hospital, Taipei, Taiwan, China

${ }^{6}$ Faculty of Medicine, School of Medicine, National Yang-Ming University, Taipei, Taiwan, China

*Corresponding author

Received 3 September 2009; revised 14 September 2009; accepted 15 September 2009.

\begin{abstract}
The purpose of this study is to explore whether it is worthwhile to launch a routine diabetic retinopathy (DR) screening for blindness prevention among Chinese type 2 diabetes from different perspective based on the population-based study in Kinmen, Taiwan. A total of 971 community dwelling adults previously diagnosed with type 2 diabetes in 1991-1993 underwent DR screening in 1999-2002 by a panel of ophthalmologists using on-site indirect ophthalmoscopy and 45-degree color fundus retinal photographs. The cost-benefit analysis is used to evaluate the DR screening. In terms of benefit-cost ratio, the different screening programs for DR could save New Taiwan Dollars (NTD) from 14.38 to 36.83 in discounted costs for each dollar incurred in different screening years from the societal viewpoint for Taiwan and save NTD from 0.81 to 1.80 in different screening years from health care payer's perspective. The average estimate of willingness-to-pay to translate into benefit yields NTD from 937.8 to 4,689 benefits per case due to DR screening in different screening years during 10-year follow-up. The net present value of the DR screening were NTD from $-167,318$ to $-307,251.2$ in different screening years. In conclusion, it is worthwhile to initial a routine DR screening of Chinese type 2 diabetes for blindness prevention from the societal perspective but not from consumer decision based on the willingness-to-pay perspective.
\end{abstract}

Keywords: Type 2 Diabetes; Diabetic Retinopathy; Cost-Benefit Analysis; Population-Based Study

\section{INTRODUCTION}

In developed countries, diabetic retinopathy (DR) is a major microvascular disease and is associated with increased visual impairment in type 2 diabetics [1]. In Taiwan, previous community-based studies showed that the prevalence and annual incidence density of DR were an estimated $15 \%$ to $45 \%$ and $6.62 \times 10^{-2}$ year $(95 \% \mathrm{CI}$ : $5.36 \times 10^{-2} /$ year $-8.06 \times 10^{-2} /$ year), respectively [2,3]. Since the average time from development of no DR to blindness is approximately 26.5 years in persons with type 2 diabetes, assessing the progression of DR by screening is a worthwhile preventive measure [4].

Whether screening for DR is worthwhile is also contingent on whether subjects are willing to pay the eye screening program that would decrease the risk of blindness. Benefit due to the reduction of severe complication in cost-benefit analysis is often measured by the human capital approach for which the value of eye screening program is measured by its effect on the patient's sight-time earning. According to welfare economic theory, the benefit to an individual of a service or an intervention is defined as that individual's maximum willingness to pay (WTP) for the service or intervention [5]. WTP is a contingent valuation and involves using a hypothetical survey to directly ask individuals the maximum amount they are willing to pay for the commodity in question $[6,7]$. Due to the medical resources are limited, the government is in a dilemma about 
whether it is necessary to popularize the eye screening programs among type 2 diabetes into a nationwide program. Our previous study have showed that degree of DR was the independent factor affecting WTP values in DR screening among community-dwelling adults with type 2 diabetes [8]. The purpose of this study is further to conduct a cost-benefit analysis to determine whether it is worthwhile to launch eye screening program for blindness prevention among population-based adults diagnosed with type 2 diabetes in Kinmen, Taiwan.

\section{METHODS}

\subsection{Study Design and Subjects}

The procedures for cost-benefit analysis of DR screening among community dwelling patients with type 2 diabetes in 1999-2003 showed in Figure 1. Data used in this study were derived from a population-based screening for type 2 diabetes targeted to subjects aged 30 years or more in Kinmen, Taiwan, between January 1991 and December 1993. Details of the study design and execution have been described in full elsewhere [9]. Identification for type 2 diabetes was based on the WHO 1999 definition [10], that is, subjects with FPG $\geq 126 \mathrm{mg} / \mathrm{dl}$ or 2-hr postload glucose concentration $\geq 200 \mathrm{mg} / \mathrm{dl}$ were defined as having type 2 diabetes. Subjects with a history of type 2 diabetes and who received medication were defined as known cases. A total of 1,123 cases of type 2 diabetes aged 30 and over were found based on the population survey carried out by the Yang-Ming Crusade, which was organized from the medical students of the National Yang-Ming University, Taipei, Taiwan. The screened diabetic subjects then were referred to the region hospital for further treatment and follow-up routinely. Of 1,123 subjects with type 2 diabetes, 152 emigrated or died between 1994 and 1998. After excluding these subjects, the remaining 971 had the uptake of fundus check-up annually since 1999. A panel of population-based follow-up screening of DR was then conducted annually from 1999 to 2002. These 971 participants were invited to receive eye screening by invitation letter or calls. Based on the eye screening results, different treatment strategies were used, that is, routine follow-up for patients with mild or moderate DR and laser photocoagulation for patients with severe DR. In addition, informed consent was obtained from all participants before the investigation began. Access to personal records was approved by the hospital human subjects review board at Cheng-Hsin General Hospital, Taipei, Taiwan.

We initiated a DR screening program after six years of mass screening because Kinmen is an offshore island from Taiwan lacking medical resources. Here, DR screening requires mobilizing manpower and equipment, coordinating between clinical personnel and field work, and transporting to the island. By 1999, a team for DR screening was successfully organized, including 4 welltrained senior ophthalmologists from Veterans General Hospital, Taipei, 4 clinical nurses, and 20 medical students from the Yang-Ming Crusade.

\subsection{Screening and Diagnosis for Diabetic Retinopathy}

The diagnosis of DR was based on the on-site direct and indirect ophthalmoscopic examination and the single-field fundus photographs that analyzed later. On-site screening was conducted by two ophthalmologists using direct and indirect ophthalmoscopy after pupil dilatation with topical $0.5 \%$ mydriacyl. The graders wrote down the diagnosis and marked the findings in the record. Then the subjects were taken one 45-degree color fundus photography with Polaroid 600 films (Polaroid, Netherland) centered at macula in each eye using Topcon fundus camera (TRC-50VT, Tokyo, Japan). The single-field photographs were then printed out and filed. Grading of the photographs was done by two well-trained senior ophthalmologists beginning no later than one month after the screening. The final grading of the DR depends on the summed interpretation of the photographs and the recorded ophthalmoscopic gradings. From the Retinopathy Disease Severity Scale, diabetic subjects were classified according to the most severe changes in the worse eye [11]. In addition, legal blindness was defined as a best-corrected visual acuity of $0.1(6 / 60)$ or worse in the better eye [8].

A pilot study was performed in 50 randomly selected type 2 diabetic patients to set up the consistent diagnosis of DR among ophthalmologists. The kappa statistic of 0.73 (95\%CI: 0.48-0.98) between the observers revealed a good interobserver reliability.

\subsection{Cost-Benefit Analysis of Screening for Diabetic Retinopathy}

\subsubsection{Markov Decision Model of Screening for Diabetic Retinopathy}

In this study, the cost-benefit analysis tool of screening for DR among subjects with type 2 diabetes was based on TreeAge software (DATA 3.5, Tree-Age, Inc., Williamstown MA) for medical decision analysis. A decision analysis using the Markov Decision Model was constructed to compare different screening regimes for DR with no screening group (see Figure 2). The assumption of no screening group was that except eye screening, diabetic patients still received routine medical care until they become blind. According to the theory of stochastic process, the Markov chain model is determined by both the initial state and the transition matrix. The model starts from the decision to screen or not to screen and the overall expected value is based on expected values of end nodes rather than all nodes. For each decision, there are six states of disease natural his 
Table 1. Cost assumptions, willingness-to-pay value, transition probabilities in decision analysis of screening for diabetic retinopathy.

\begin{tabular}{lc}
\hline Parameter & Value \\
\hline Annual direct cost (NT dollars) & \\
Screening cost & \\
Drug cost $^{2}$ & 2,298 \\
Regular clinics fee $^{3}$ & 10,857 \\
Laser photocoagulation $^{4}$ & 509 \\
Vitrectomy & 10,970 \\
Total & 10,840 \\
\hline
\end{tabular}

Annual indirect cost (NT dollars)

Gross Domestic Product, GDP 452,168

\begin{tabular}{ll}
\hline Willingness-to-pay value(NT dollars) [8] & \\
No DR & $440.1 \pm 331.6$ \\
NPDR & $450.0 \pm 298.8$ \\
PDR & $683.3 \pm 285.5$ \\
Legal blindness & $822.2 \pm 192.2$ \\
Total & $468.9 \pm 327.7$ \\
\hline
\end{tabular}

Annual transition probability (\%) [4]

$\begin{array}{ll}\text { No DR } \rightarrow \text { Mild NPDR } & 7.37\end{array}$

$\begin{array}{ll}\text { Mild NPDR } \rightarrow \text { Moderate NPDR } & 19.37\end{array}$

Moderate NPDR $\rightarrow$ Severe NPDR $\quad 17.41$

$\begin{array}{ll}\text { Severe NPDR } \rightarrow \text { PDR } & 28.95\end{array}$

PDR $\rightarrow$ Legal blindness $\quad 21.10$

\footnotetext{
${ }^{1}$ Screening cost includes clinician's fee, vision examination, pupil dilation, slit lamp contact mirror funduscopy, funduscopic exam, HbA1c, SMA-12 test, and manpower cost.

${ }^{2}$ According to the drug usage distribution from Taiwanese Association of Diabetes Educators (TADE) study in 2004 and the payment of National Health Insurance.

${ }^{3}$ Regular clinics fee includes clinician's fee and pharmacist's fee

${ }^{4}$ Laser photocoagulation cost includes panretinal photocoagulation, two fundus color photos, and fluorescein angiography (FAG).

${ }^{5}$ Vitrectomy cost includes vitretomy, two fundus color photos, and fluorescein angiography (FAG).
}

tory of DR including NDR, mild NPDR, moderate NPDR, severe NPDR, PDR, and blindness. The initial state distribution is based on the results of the present study. Transition probabilities from one state to another representing the disease natural history of DR were derived from our empirical estimation, that is, the annual transition probabilities for each stage to the next are as follows: mild NPDR to moderate NPDR $19.37 \%$, moderate NPDR to severe NPDR $17.41 \%$, severe NPDR to PDR 28.95\%, and PDR to blindness 21.10\% [4]. For each scenario, we calculated the expected probability of patients aggregate experience that is accumulated in each state during 10-year follow-up.

\subsubsection{An Empirical Survey for the Estimation of Cost and Willingness to Pay}

Costs in the present study include direct and indirect cost. Direct costs include cost of DR screening, drug cost, cost of regular clinic fee, and treatment cost (for example, laser photocoagulation and surgery). Indirect cost includes only productivity loss of the patient because of time taken off work for treatment. The average time taken off work for treatment depends on the professions' opinion. In addition, WTP was assessed by the following question: "What is the most price that you would be willing to pay for routine screening for DR that reduces the risk of fully blindness?” The WTP amounts 
Table 2. Cost-benefit analysis using the human-capital approach of different screening programs for diabetic retinopathy.

\begin{tabular}{|c|c|c|c|}
\hline & Screening group & Non-screening group & Net cost \\
\hline \multicolumn{4}{|l|}{ Annual screening } \\
\hline \multicolumn{4}{|l|}{ Direct cost } \\
\hline Screening cost & 21,900 & 0 & 21,900 \\
\hline Other cost & 113,403 & 131,219 & $-17,816$ \\
\hline Total & 135,303 & 131,219 & 4,084 \\
\hline Indirect cost & 36,704 & 333,911 & $-297,207$ \\
\hline Total (Direct + Indirect cost) & 172,007 & 465,130 & $-293,123$ \\
\hline \multicolumn{4}{|l|}{ Biennial screening } \\
\hline \multicolumn{4}{|l|}{ Direct cost } \\
\hline Screening cost & 10,950 & 0 & 10,950 \\
\hline Other cost & 116,216 & 131,219 & $-15,003$ \\
\hline Total & 127,166 & 131,219 & $-4,053$ \\
\hline Indirect cost & 70,435 & 333,911 & $-263,476$ \\
\hline Total (Direct + Indirect cost) & 197,601 & 465,130 & $-267,529$ \\
\hline \multicolumn{4}{|l|}{ 3-yearly screening } \\
\hline \multicolumn{4}{|l|}{ Direct cost } \\
\hline Screening cost & 7,300 & 0 & 7,300 \\
\hline Other cost & 118,832 & 131,219 & $-12,387$ \\
\hline Total & 126,132 & 131,219 & $-5,087$ \\
\hline Indirect cost & 107,629 & 333,911 & $-226,282$ \\
\hline Total (Direct + Indirect cost) & 233,761 & 465,130 & $-231,369$ \\
\hline \multicolumn{4}{|l|}{ 4-yearly screening } \\
\hline \multicolumn{4}{|l|}{ Direct cost } \\
\hline Screening cost & 5,475 & 0 & 5,475 \\
\hline Other cost & 121,199 & 131,219 & $-10,020$ \\
\hline Total & 126,674 & 131,219 & $-4,545$ \\
\hline Indirect cost & 144,729 & 333,911 & $-189,182$ \\
\hline Total (Direct + Indirect cost) & 271,403 & 465,130 & $-193,727$ \\
\hline \multicolumn{4}{|l|}{ 5-yearly screening } \\
\hline \multicolumn{4}{|l|}{ Direct cost } \\
\hline Screening cost & 4,380 & 0 & 4,380 \\
\hline Other cost & 123,330 & 131,219 & $-7,889$ \\
\hline Total & 127,710 & 131,219 & $-3,509$ \\
\hline Indirect cost & 180,479 & 333,911 & $-153,432$ \\
\hline Total (Direct + Indirect cost) & 308,189 & 465,130 & $-156,941$ \\
\hline
\end{tabular}


Table 3. The benefit-cost ratio estimates of different screening programs for diabetic retinopathy.

\begin{tabular}{lllcc}
\hline & \multicolumn{3}{c}{ Benefit-cost ratio } \\
\cline { 2 - 5 } & \multicolumn{2}{c}{ Payer’s perspective } & \multicolumn{2}{c}{ Society perspective } \\
\hline Annual screening & 0.81 & $(17,816 / 21,900)$ & 14.38 & $((17,816+297,207) / 21,900)$ \\
Biennial screening & 1.37 & $(15,003 / 10,950)$ & 25.43 & $((15,003+263,476) / 10,950)$ \\
3-yearly screening & 1.70 & $(12,387 / 7,300)$ & 32.69 & $((12,387+226,282) / 7,300)$ \\
4-yearly screening & 1.83 & $(10,020 / 5,475)$ & 36.38 & $((10,020+189,182) / 5,475)$ \\
5-yearly screening & 1.80 & $(7,889 / 4,380)$ & 36.83 & $((7,889+153,432) / 4,380)$ \\
\hline
\end{tabular}

Screening for type 2 diabetes (1991-1993)
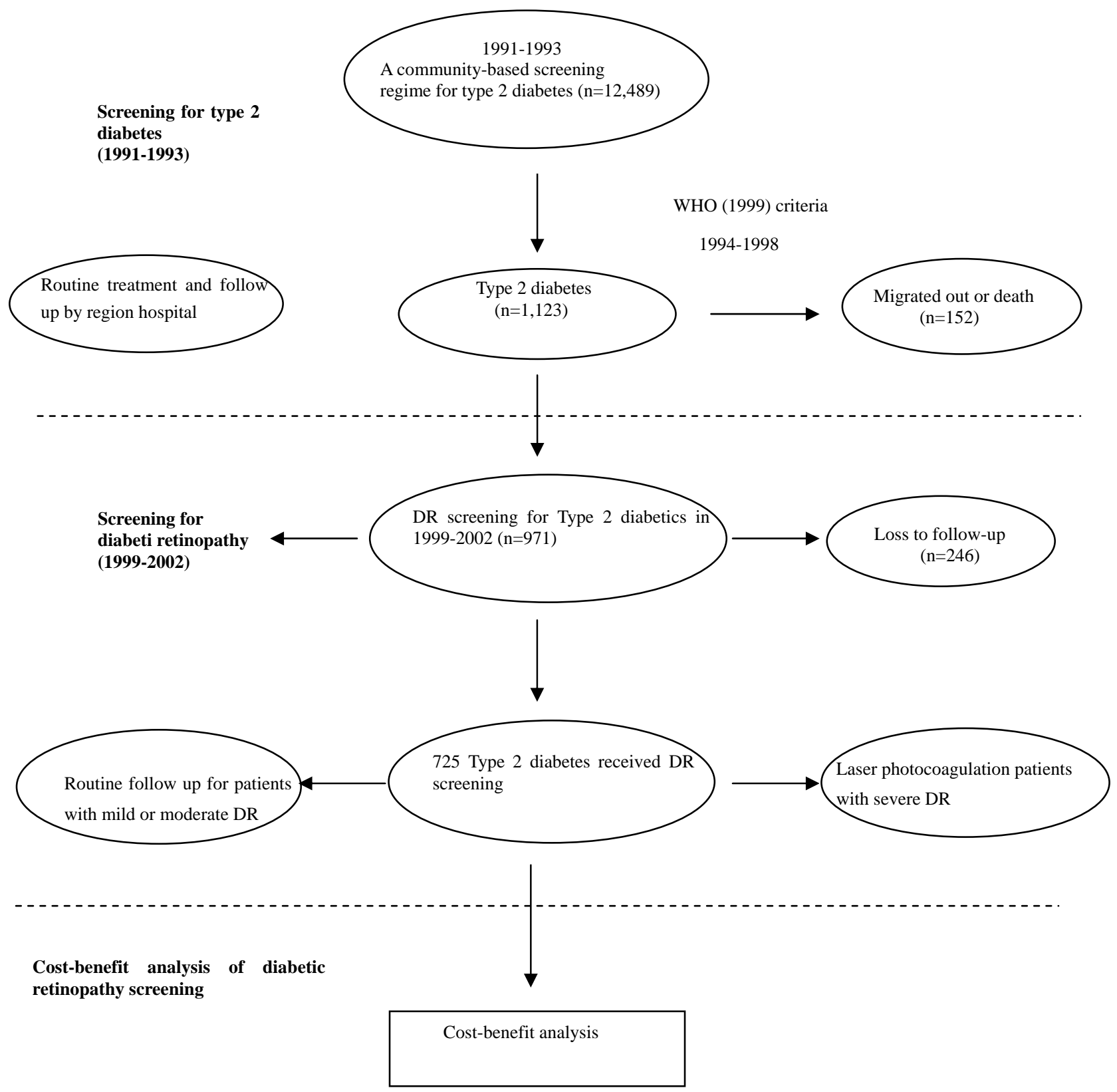

Figure 1. The procedure of cost-benefit analysis of screening for diabetic retinopathy among type 2 diabetics in Kinmen. 


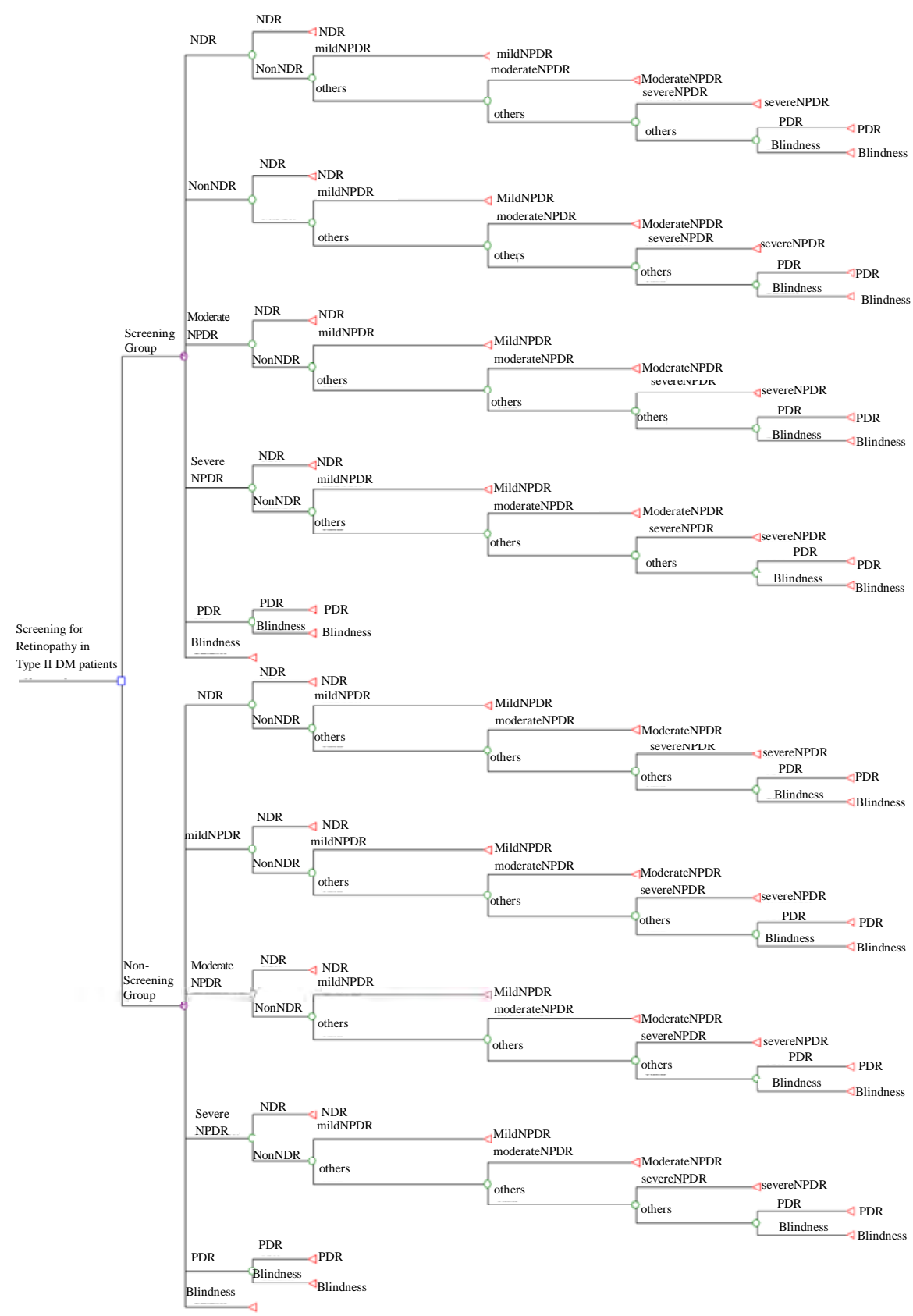

Figure 2. Markov decision model for two options, screening and non-screening for diabetic retinopathy.

for a routine screening for DR were elicited by discrete-choice, that is, subjects were presented a single price for a screening program that would yield a specified health change. Subjects either accept or reject the price. By randomly varying the price across a number of different subsamples, the mean WTP could be estimated [8]. To maintain consistency of interview quality, all information on WTP measurements was also collected by one well-trained interviewer. All costs are expressed as New Taiwan Dollars (NTD).

\subsubsection{Cost-Benefit Analysis and Discount Rate}

Using the human-capital approach, net cost (saving) for different screening programs of DR, taking direct cost and indirect cost into account, was calculated. Benefit-cost ratios were calculated as the reduction blindness costs divided by the cost of the screening programs. Using WTP approach, net present value (NPV) was also calculated on the basis of the total benefit (calculated by the WTP method) minus screening cost of DR. To take time preference into account, that is, receiving benefit 
earlier and incurring cost later, we discounted all costs and benefits to the present value at 5\% annually.

\section{RESULTS}

The annual direct cost, annual indirect cost, WTP value, and annual transition probability in decision analysis of DR screening are shown in Table 1. Direct costs include screening cost, drug cost, regular clinics' fees, laser photocoagulation, and vitrectomy. Indirect cost represents lost productivity according to patient's disease state, estimated using the Gross Domestic Product (GDP) value in 2004.

Table 2 shows total discounted direct costs and indirect costs using the human capital approach. Annual screening, biennial screening, 3-yearly screening, 4-yearly screening, and 5-yearly screening regimens could save NTD17,816, NTD15,003, NTD12,387, NTD10,020, and NTD7,889 per case in discounted direct costs (except screening cost), respectively. From payer's perspective, the discounted net total direct costs for DR screening program were NTD 4,084, NTD-4,053, NTD-5,087, NTD-4,545, and NTD3,509 for annual screening, biennial screening, 3-yearly screening, 4-yearly screening, and 5-yearly screening, respectively. The discounted indirect costs saved per case by DR screening program were NTD297,207, NTD 263,476, NTD226,282, NTD189,182, and NTD153,432 for annual screening, biennial screening, 3-yearly screening, 4-yearly screening, and 5-yearly screening, respectively. This yield NTD293,123 (annual screening), NTD267,529 (biennial screening), NTD231,369 (3-yearly screening), NTD 193,727 (4-yearly screening), and NTD156,941 (5-yearly screening) net saving per case due to DR screening program from the societal perspective.

As Table 3 shows, DR screening programs could save NTD14.38 (annual screening), NTD25.43 (biennial screening), NTD32.69 (3-yearly screening), NTD36.38 (4-yearly screening), and NTD36.83 (5-yearly screening) from the societal viewpoint and save NTD0.81 (annual screening), NTD1.37 (biennial screening), NTD1.70 (3yearly screening), NTD1.83 (4-yearly screening), and NTD1.80 (5-yearly screening) in discounted costs for each NTD dollar incurred in DR screening programs from health care payer's perspective.

The average estimate of WTP in order to reduce blindness as shown in Table 1 is NTD468.9. Translating this figure into benefit yields NTD4,689 (annual screening), NTD2,344.5 (biennial screening), NTD1,406.7 (3yearly screening), NTD1,172.3 (4-yearly screening), and NTD 937.8 (5-yearly screening) benefit per case due to DR screening during 10-year follow-up. The NPV of the screening programs, taking indirect cost into account, were NTD-167,318, NTD-195,256.5, NTD-232,354.3, NTD-270,230.7, and NTD-307,251.2 of annual screening, biennial screening, 3-yearly screening, 4-yearly screening, and 5-yearly screening, respectively.

\section{DISCUSSION}

\subsection{Implications of Cost-Benefit Analysis for Diabetic Retinopathy Screening}

In Taiwan, few population-based studies have attempted to quantify the cost and benefit of DR screening programs. The present study uses cost-benefit analysis to assess whether a DR screening program against nonscreening group is worthwhile in Taiwan from different perspectives. The results indicate that indirect costs play an important role in the evaluation of the DR screening program. Annual screening program could save the most (NTD297,207) per case in discounted indirect costs compared with non-screening group. From health care payer's perspective, the discounted net cost for annual screening was NTD4,084 per case. This indicates that the benefit from the annual screening program of DR can not outweigh the cost incurred in the DR screening program from health care payer's perspective. Taking indirect cost into account, the NTD293,123 net saving per case suggest the annual screening program is rather worthwhile from the societal perspective.

In addition, using the WTP approach, the present study shows it is not worthwhile to have screening for DR from the perspective of WTP due to the negative result of NPV value. It should be noted that the WTP approach is a contingent-valuation method that reflects consume surplus of getting DR screening. Since the mean estimate of WTP (NTD468.9) for the DR screening program was far below the current expense for direct costs of DR screening (NTD2,190) per case. This suggests that they could not get any surplus from the purchase of screening program. This accounts for why the results of NPV are negative. If the estimate of WTP is raised to NTD2,500, this means that if people have to pay only NTD2,190 for benefits they value at NTD2,500 then they get a surplus of NTD310 from the purchase of screening for DR. Results from the WTP suggest that the amount diabetics in Kinmen are willing to pay for the screening program is lower than the benefit they value. In terms of consumer decision based on the perspective of WTP, it may not be worthwhile to launch a DR screening program.

\subsection{The Efficiency and Advantage for Routine Diabetic Retinopathy Screening}

The surveillance and treatment of diabetes-related complications should be part of routine care in all type 2 diabetes [4]. The benefits of DR screening rest on the additional time patients have to obtain treatment. If one wishes to reduce the loss of vision associated with DR, 
then performing the screening is more important than the type of screening used [12,13]. During the first year of DR screening, savings associated with preventing one single case of blindness could cover the cost of the screening program [12]. The threat of blindness is less severe in type 2 diabetes because DR progresses more slowly than in type 1 diabetes. Although the eye care program saved 21 sight-years [14], it was less efficient in patients with type 2 diabetes.

The optimal screening interval is determined by the disease natural history with regard to the screening policy [4]. Many evidence-based studies have showed that screening for and treating DR is extremely efficiency and cost-effective. From the health insurer's viewpoint, routine screening and treatment of eye disease in diabetic patients cost USD 3190 per QALY saved [15]. Duration of blindness drops by 0.48 and 0.13 years with increase in year of onset of the disease whereas effectiveness decreases in type 2 diabetic patients [16]. Prevention screening programs aimed at improving eye care for type 2 diabetic patients results in both highly cost-effective health care and substantial federal budgetary savings $[15,17]$. In addition, our previous studies have demonstrated that annual DR screening is the most effective and efficient screening schedule for reducing blindness compared to other screening intervals [4,17]. To take both cost and efficacy into consideration, many organizations such as the National Committee for Quality Assurance, through Health Employer Data and Information Set (HEDIS) measures also recommends that annual eye examinations be used not only as a general guideline, but also as a quality standard in all patients with diabetes [18]. Better diabetes management such more strict adherence by diabetic patients and ophthalmologists to best practice guidelines could have enormous protective impacts in cases of visual loss caused by DR [19].

\subsection{Methodological Considerations}

From the methodological viewpoint, although using a population-based follow-up study design could reduce selection bias and increase statistical power, using primary information and calculating both direct and indirect costs help us estimate the true benefit of DR screening more closely than which has been possible before. There are still some critiques raised from this study. First, only three-year follow-up period, we could not have enough sample size to predict all of the effects of DR screening on disease variations. Second, we did not explicitly consider the sensitivity and specificity of the DR screening tests. Previous studies demonstrated that indirect ophthalmoscopy performed by ophthalmologists has a sensitivity of approximately 85\% [20], but this may approach $100 \%$ with newer slit lamp biomicroscopic techniques [21]. Retinal photography, an alternative detection method for DR among diabetic patients, has an overall sensitivity of approximately 85\% [20]. That means the accuracy of DR diagnosis could be accepted. Third, although the Kappa value for the agreement of interobserver reliability seemed acceptable [22], non-differential misclassification-bias identification still could have occurred. Fourth, we did not estimate the influence of covariates such as duration of type 2 diabetes or HbA1c level . Because severe and very severe NPDR without macular edema is associated with high risk of progression to PDR, $10-50 \%$ of those with type 2 diabetes and this level of NPDR will develop PDR within 1 year [23]. Further long-term studies should be conducted to clarify whether patients with better glycemic control or in an early stage of DR could benefit from less frequent screening intervals. Finally, it should be noted that the estimates used in this analysis were based on relatively small samples, that is, the aggregate estimates may reflect a reasonable population, but not all Chinese with type 2 diabetes. Further study of those inadequately represented is needed.

\section{CONCLUSIONS}

In conclusion, this study revealed that it is worthwhile to launch a routine DR screening program of Chinese type 2 diabetes for blindness prevention from the societal perspective but not from consumer decision based on the perspective of WTP.

\section{ACKNOWLEDGEMENTS}

This study was also supported by the grants from the National Science Council (NSC-95-2314-B-350-002-MY3) and (NSC-98-2314-B-350002-MY3).

\section{REFERENCES}

[1] Sanchez-Thorin, J.C. (1998) The epidemiology of diabetes mellitus and diabetic retinopathy. Intern Ophthalmol Clin, 38(2), 11-18.

[2] Chen, M.S., Kao, C.S., Chang, C.J., Wu, T.J., Fu, C.C. and Chen, C.J. (1992) Prevalence and risk factors of diabetic retinopathy among non-insulin-dependent diabetic subjects. Am J Ophthalmol, 114(6), 723-730.

[3] Tung, T.H., Chen, S.J., Liu, J.H., et al. (2005) A community-based follow-up study on diabetic retinopathy among type 2 diabetics in Kinmen. Eur J Epidemiol, 20(4), 317-323.

[4] Tung, T.H., Chen, S.J., Shih, H.C., et al. (2006) Assessing the natural course of diabetic retinopathy: A population-based study in Kinmen. Taiwan, Ophthalmic Epidemiol, 13(5), 327-333.

[5] Bala, M.V., Mauskopf, J.A. and Wood, L.L. (1999) Willingness to pay as a measure of health benefits. Pharmacoeconomics, 15(1), 9-18. 
[6] Jampel, H.D., Schwartz, G.F., Robin, A.L., Abrams, D.A., Johnson, E. and Miller, R.B. (2003) Patient preferences for eye drop characteristics: A willingness-to-pay analysis. Arch Ophthalmol, 121(4), 540-546.

[7] Olsen, J.A. and Smith, R.D. (2001) Theory versus practice: A review of "willingness-to-pay" in health and health care. Health Econ, 10(1), 39-52.

[8] Shih, H.C., Chou, P., Chen, S.J., et al. (2007) A community-based study of the willingness to pay associated with screening for diabetic retinopathy among type 2 diabetes in Kinmen, Taiwan. J Epidemiol, 17(6), 186-193.

[9] Chou, P., Liao, M.J., Kuo, H.S., Hsiao, K.J. and Tsai, S.T. (1994) A population survey on the prevalence of diabetes in Kin-Hu, Kinmen. Diabetes Care, 17(9), 1055-1058.

[10] World Health Organization (1999) Definition, diagnosis and classification of diabetes mellitus and its complications: Report of a WHO consultation. Part 1. Diagnosis and classification of diabetes mellitus. Geneva, World Health Organization.

[11] Wilkinson, C.P., Ferris $3^{\text {rd }}$, F.L., Klein, R.E., et al. (2003) Proposed international clinical diabetic retinopathy and diabetic macular edema disease severity scales. Ophthalmology, 110(9), 1677-1682.

[12] García-Serrano, J.L., Serrano-Laborda, D., López-Pozas, M., Cabello-Aparicio, C. and Castro-Rosales, L. (2007) The Granada diabetic retinopathy study: Direct screening of 8,244 patients. I Arch Soc Esp Oftalmol, 82(11), 681-689.

[13] Harvey, J.N., Craney, L., Nagendran, S. and Ng, C.S. (2006) Towards comprehensive population-based screening for diabetic retinopathy: Operation of the North Wales diabetic retinopathy screening programme using a central patient register and various screening methods. J Med Screen, 13(2), 87-92.

[14] Ackerman, S.J. (1992) Benefits of preventive programs in eye care are visible on the bottom line. A new nationwide effort to improve eye care for people with diabetes gets backing from a study on the cost-effectiveness of screening for retinopathy. Diabetes Care, 15(4), 580-581.

[15] Javitt, J.C. and Aiello, L.P. (1996) Cost-effectiveness of detecting and treating diabetic retinopathy. Ann Intern Med, 124(1 Pt 2), 164-169.

[16] Polak, B.C., Crijns, H., Casparie, A.F., et al. (2003) Cost-effectiveness of glycemic control and ophthalmological care in diabetic retinopathy. Health Policy, 64(1), 89-97.

[17] Tung, T.H., Shih, H.C., Chen, S.J., et al. (2008) Economic evaluation of screening for diabetic retinopathy among Chinese type 2 diabetics: A community-based study in Kinmen, Taiwan. J Epidemiol, 18(5), 225-233.

[18] Health Employer Data and Information Set (1997) Version 3.0. Washington, DC: National Committee for Quality Assurance, 2.

[19] Sloan, F.A., Grossman, D.S. and Lee, P.P. (2009) Effects of receipt of guideline recommended care on onset of diabetic retinopathy and its progression. Ophthalmology, 116(8), 1515-1521.

[20] Klein, R., Klein, B.E, Neider, M.W., Hubbard, L.D., Meuer, S.M. and Brothers, R.J. (1985) Diabetic retinopathy as detected using ophthalmoscopy, a nonmydriatic camera and a standard fundus camera. Ophthalmology, 92(4), 485-491.

[21] Ekoe, J.M., Zimmet, P. and Williams, R. (2001) The epidemiology of diabetes mellitus: An international perspective. John Wiley \& Sons, Inc.

[22] Byrt, T. (1996) How good is that agreement. Epidemiology, 7(5), 561.

[23] Aiello, L.P., Gardner, T.W., King, G.L., et al. (1998) Diabetes retinopathy. Diabetes Care, 21(1), 143-156. 